\title{
Opinion dynamics and decision of vote in bipolar political systems
}

\author{
Filippo Caruso ${ }^{3}$ and Paolo Castorina ${ }^{2,1}$ \\ ${ }^{1}$ Dept. of Physics, University of Catania and ${ }^{2}$ INFN, Sezione di Catania, \\ Via S. Sofia 64, I-95123, Catania, Italy \\ ${ }^{3}$ Scuola Superiore di Catania, Via S. Paolo 73, 95123 Catania, Italy \\ filippo.caruso@ct.infn.it, paolo.castorina@ct.infn.it
}

(Dated: 13th August 2018)

\begin{abstract}
A model of the opinion dynamics underlying the political decision is proposed. The analysis is restricted to a bipolar scheme with a possible third political area. The interaction among voters is local but the final decision strongly depends on global effects such as, for example, the rating of the governments. As in the realistic case, the individual decision making process is determined by the most relevant personal interests and problems. The phenomenological analysis of the national vote in Italy and Germany has been carried out and a prediction of the next Italian vote as a function of the government rating is presented.
\end{abstract}

Keywords: disordered systems, opinion dynamics

\section{INTRODUCTION}

The study of complex systems entered social science in order to understand how self-organization, cooperative effects and adaptation arise in social communities. In this context the use of simple automata or dynamical models often elucidates the underlying dynamics of the observed behaviour (1-13).

In particular some models have been proposed to describe the opinion dynamics and the final decision of a community of voters $(3,5-13)$.

These models focus on the self-organization resulting from a local dynamics, which represents the mutual influence, based on two simple properties:

i) individuals are more likely to interact with others who already share many of their opinions;

ii) interaction increases the number of features that individuals share.

However, a deeper analysis of the process which leads to the final decision in a political vote requires an improvement of the proposed models.

This can be done in a mathematical way by considering the application of the game theory to political problems 14] or, more simply, by introducing in the decision process other important global effects such as, for example, the Government policy and/or the propaganda, which are usually neglected.

In this paper we propose a model toward a more realistic description of the opinion dynamics underlying the choice of the voters. The analysis is restricted to a bipolar scheme with a possible third political area, but it can be generalized to the case of many political parties. This initial simplification helps to clarify the model and, anyway, it applies to many European and non European countries.

The model is based on the following points to be formalized and discussed in details later on:

1) There are initially three different groups of voters: the right Coalition (RC), the left Coalition (LC) and a central group (CG). The voters in the $\mathrm{RC}$ or $\mathrm{LC}$ do not change their opinion: they represent the cores of the political bipolar scheme.

2) The individuals of the CG interact with each other and with the individuals of the RC and of the LC. At the end of the dynamical process there are three groups: majority, minority and the others.

3) The final decision of the single CG voter is based on his/her opinions on the arguments that he/she considers more relevant.

4) The mutual local influence among voters is modified by the global degree of satisfaction/dissatisfaction with respect to the Government.

The scheme (1-4) is, of course, an extremely simplified version of the real processes. For example the possibility that each voter (LC, RC, CG) decides not to vote (abstentionism) is not taken into account in the dynamics and this implies that the abstentionism is proportionally distributed among the different groups.

However the model has many steps of analysis,it requires the application of general techniques of the complex systems and, as we shall see, it is able to give some interesting indications on the political dynamics.

The paper is organized as follows: the cognitive political model and the social-political interaction are described in Sec. 1; in Sec. 2 we set the criterion of political decision; in Sec. 3 the approximations introduced in the numerical simulations are discussed; the phenomenological analysis of the political vote in Italy and Germany and the prediction for the next Italian vote are carried out in Sec. 4; Sec. 5 is devoted to the conclusions and outlooks. 


\section{THE COGNITIVE POLITICAL MODEL}

\section{Model general structure}

In simulating the opinion dynamics toward a final political vote one has to generalize the available models of social interaction.

In the Axelrod model [7, 8] of the cultural evolution the interaction is limited to an imitation process in which the agents adapt cultural traits stochastically from each other with a bias toward similar agents. The interesting final result of the evolution is the diversity: there are different cultural domains.

In the bounded confidence model [9, 10, 12], more oriented to the analysis of voting patterns, each voter possesses a single real valued opinion. When two voters interact, they average their opinions only if the opinion difference is within an external pre-fixed threshold otherwise there is no interaction. Also in this case, as in the previous models, the system breaks up into distinct opinion clusters.

However, in the analysis of the political opinion dynamics there is, ab initio, a community with a nonnegligible heterogeneity and the selfish individual convictions play a crucial role not only in the interaction among individuals but also in the degree of influence of global effects (governments, mass media,social shocks [15, 16]) on the single voter.

In particular,in a bipolar political system, the number of votes of the two most important (left and right) coalitions depends upon an almost constant core of voters, who do not change their opinion, and on the individuals with less strong political convictions (the CG) who decide on the basis of a personal political analysis.

More precisely, in the political decision process each individual is in front of many questions of social importance (the contexts) and he/she has to evaluate the possible alternative choices.

This analysis, based on a personal mental representation of the validity of the different alternatives, evolves according to the interaction with the other members of the community and according to the global influence.

As a result of this process, a restricted number of more relevant concepts emerges: in the individual decision making mechanism there is a simplification with respect to the social-political complexity and a "dimensional reduction" to the most relevant aspects.

The previous considerations can be formalized by following the interesting cognitive model proposed in ref. 13. that, with some peculiar modifications (see later), is a good starting point to investigate the opinion dynamics toward a realistic simulation of the final political vote.

In ref. 13] one considers $I$ agents who divide the world into a number $X$ of contexts in which they evaluate alternative possibilities (scenarios) according to their personal opinions. The alternative scenarios are characterized by their objective attributes $\boldsymbol{a}=\left\{a_{1}, \ldots, a_{D}\right\}$. A crucial assumption of the model is that the Agent $i$ 's theoretical payoff from the realization of a possible scenario $\boldsymbol{a}$ in context $x$ is posited to be a linear function

$$
\pi_{i}^{(x)}(\boldsymbol{a})=\boldsymbol{\omega}_{i}^{(x)} \cdot \boldsymbol{a}
$$

where $\boldsymbol{\omega}_{i}^{(x)}$ is the agent's context vector, reflecting actual circumstances of the context and the agent's personal opinions. For each agent there are $X$ context vectors each of dimension $D$, which are assumed fixed in the model.

An agent does not know his/her context vectors explicitly as this would require a detailed understanding of the effect of all attributes on his payoffs. However, by collecting experience on choices he/she has made previously, he/she learns to approximate the payoffs using an appropriate mental representation. The mental representation is built around the world's $K$ "most important degrees of freedom", constituting the agent's Concepts. One assumes again that the approximate payoff that the agent "computes" directly is linear

$$
\tilde{\pi}_{i}^{(x)}(\boldsymbol{a})=\tilde{\boldsymbol{\omega}}_{i}^{(x)} \cdot \boldsymbol{a}
$$

with

$$
\tilde{\boldsymbol{\omega}}_{i}^{(x)}=\sum_{\mu=1}^{K} v_{i \mu}^{(x)} \gamma_{i \mu}
$$

the agent's approximate context vector.

By eq.(3), $\tilde{\boldsymbol{\omega}}_{i}^{(x)}$ is decomposed using mental weights $v_{i \mu}^{(x)}$ in a reduced subspace of dimension $K$ and a number $K$ of concept vectors, $\left\{\gamma_{i \mu}\right\}_{\mu=1}^{K}$, assumed normalized $\left|\gamma_{i \mu}\right|=1$, which the agent uses to evaluate alternatives.

Due to the reduction of dimensionality, $K<D$, the approximate payoff $\tilde{\pi}_{i}^{(x)}$ deviates from the theoretical payoff $\pi_{i}^{(x)}$. The agents' goal is to find the best possible set of Concepts and mental weights which minimizes the error of the mental representation under the constraint that only $K$ Concepts can be used. The natural measure of agent $i$ 's representation error is the variance

$$
E_{i}=\sum_{x=1}^{X}\left\langle\left(\pi_{i}^{(x)}-\tilde{\pi}_{i}^{(x)}\right)^{2}\right\rangle_{x}
$$

where $\langle.\rangle_{x}$ is the average over alternatives in context $x$, that can be written as

$$
E_{i}=\sum_{x=1}^{X}\left|\boldsymbol{\omega}_{i}^{(x)}\right|^{2}-U_{i}
$$

where the agent's utility $U_{i}$ is given by 


$$
U_{i}=\sum_{\mu=1}^{K} \gamma_{i \mu} \cdot \boldsymbol{W}_{i} \cdot \gamma_{i}^{\mu}
$$

and $W_{i}$ is a positive semi-definite, $D \times D$ dimensional matrix, called the world matrix,

$$
\boldsymbol{W}_{i}=\sum_{x=1}^{X} \boldsymbol{\omega}_{i}^{(x)} \circ \boldsymbol{\omega}_{i}^{(x)}
$$

which encompasses all information about agent $i$ 's relationship to the world.

The criterion for the political decision, that will be discussed in sec. 2, is based on the minimization of $E_{i}$ which is equivalent to maximize $U_{i}$. This is the wellknown Principal Component Analysis (PCA) problem. According to this, the optimal concept vectors are provided by the $K$ most significant (largest eigenvalues) eigenvectors of $\boldsymbol{W}_{i}$ in the considered basis of the D dimensional space (see below). Thus to achieve the best possible mental representation the agent should choose his/her concept vectors according to the eigenvectors of his/her world matrix in the order of their significance.

Two important remarks are now in order:

a) In the application of the model to the political dynamics the previous considerations apply only to the agents in the $\mathrm{CG}$ (see the next subsections). The agents in the $\mathrm{RC}$ and $\mathrm{LC}$ are assumed to have fixed orthogonal concept vectors ,called $r_{\mu}$ and $l_{\mu}$ respectively, with scalar products $r_{\mu} r_{\nu}=\delta_{\mu \nu}, l_{\mu} l_{\nu}=\delta_{\mu \nu}, r_{\mu} l_{\mu}=0$. These vectors form the basis of two fixed orthogonal subspaces.

The orthogonality of the subspaces associated with the $\mathrm{LC}$ and the $\mathrm{RC}$ is also an approximation because in the real dynamics the concept vectors of the $\mathrm{LC}$ and $\mathrm{RC}$ are not always completely orthogonal (bipartisan choices). With this approximation one assumes that the bipartisan choices are irrelevant for the final political decision.

b) The identification, sorting and truncation of the degrees of freedom in the model is closely analogous to what occurs in White's Density Matrix Renormalization Group method (DMRG) 17, 18]). In the DMRG the optimally renormalized degrees of freedom turn out to be the $K$ most significant eigenvectors of the reduced density matrix of the quantum subsystem embedded in the environment with which it interacts.

\section{The socio-political interaction}

As discussed in the introduction, the opinion dynamics is due to local interactions among voters and to global political effects. The local and global interactions, related with the previous cognitive model, will be now separately analyzed.

\section{a) The local interaction}

The representation error is minimal if the agent learns to approximate his/her world matrix in the $K$ dimensional subspace spanned by the most significant eigenvectors of his/her world matrix. The final vote decision is due to the social-political network and, in our bipolar scheme,it is useful to cast context vectors into two basic categories [13]:

1) context vectors which only depend on a single agent, with a world matrix $W_{i}^{0}$

2) context vectors for agent $i$ which depend on the interaction with at least one other agent $j$.

For simplicity only pair interactions will be considered and following ref. [13] the world matrix to be used in the utility function is written as

$$
\boldsymbol{W}_{i}=\boldsymbol{W}_{i}^{0}+\sum_{j \in \mathcal{C} \mathcal{G}} c_{i j} \boldsymbol{S}_{j}+\sum_{j \in \mathcal{L C}} l_{i j} \boldsymbol{S}_{j}+\sum_{j \in \mathcal{R C}} r_{i j} \boldsymbol{S}_{j}
$$

where the total agents number is $\mathcal{N}$, the agent $i$ is in the CG (see later), the parameters $c_{i j}, l_{i j}, r_{i j}$ measure the relative strength of socio-political interactions with agent $j$ in the different groups (CG, LC, RC) and $S_{j}$ is given by

$$
\boldsymbol{S}_{j}=\sum_{\mu=1}^{K} \gamma_{j \mu} \circ \gamma_{j}^{\mu}
$$

i.e. by $S_{j}$ there is in $W_{i}$ an overlap of the concept vectors of the agents $i$ and $j$.

It is important to stress that the previous interactions are among the agents $i$ of the CG with the other agents in the same group, in the $\mathrm{LC}$ and in the $\mathrm{RC}$. Indeed, in our scheme the agents in the LC or RC do not change their opinion and their world matrices are fixed to constant $W_{i}^{0}$. Moreover the world matrix of the agents in the LC is orthogonal to the world matrix for the $\mathrm{RC}$ agents.

\section{b) The global effects}

The opinion making process in a national political vote depends only partially on the local interaction and is strongly influenced by other important elements such as the decisions of the Government and the mass media role. In order to include these effects in the dynamical process, let us introduce a set of indices, $\delta_{i}$, to describe the satisfaction of the agent $i$ with respect to the global political decisions. Again the voters in the RC and LC do not change opinion despite their satisfaction/dissatisfaction and then the satisfaction indices, $0<\delta_{i}<1$, related with the rating of the global events, are relevant only for the voters in the $\mathrm{CG}$. 
In the previous subsection, the local influence has been introduced by the political interaction matrices $c_{i, j}, l_{i, j}$, $r_{i, j}$. In the political bipolar scheme, a simple way to include the satisfaction indices, $\delta_{i}$, in the dynamics is to consider that a voter who has a positive perception of the Government actions increases the strength of its interaction with the coalition which governs while a dissatisfied voter tends to interact more with the opposition. Therefore the interaction matrices of an agent $i$ of the CG with the agents $j$ of the $\mathrm{LC}$ or of the $\mathrm{RC}$ will depend on the satisfaction index in such a way to increase the interaction with the majority and decrease the interaction with the minority or viceversa. The interaction among voters in the CG remains unchanged.

In section 3 the $l_{i, j}, r_{i, j}$ dependence on $\delta_{i}$ will be clarified.

\section{THE DECISION PROCESS}

The most important elements which determine the agent representation of the social-political system have been specified in the previous sections. Following ref. [13], one can assume that the system evolves according to a "gradient adjustment dynamics" obtained by the time evolution of the concept vectors given by

$$
\frac{\delta \gamma_{i \mu}}{\delta t} \propto \frac{\partial U_{i}}{\partial \gamma_{i \mu}}
$$

Of course, only the CG agents have a dynamical evolution because the agents in the $\mathrm{LC}$ and in the RC have fixed world matrices, $\boldsymbol{W}_{i}=\boldsymbol{W}_{i}^{0}$, and the corresponding concept vectors span orthogonal subspaces. For the agents in the CG it is reasonable to assume that without interaction they choose random concept subspaces, i.e. complete disorder, and this implies that their matrices $\boldsymbol{W}_{i}^{0}$ have a Wishart distribution (see 19,20$]$ ). After the evolution according to the best response dynamics, at the equilibrium [13], each agent of the CG decides his/her vote and one needs a well defined criterion to understand if his/her final "position" is closer to the LC or the RC or is too far from both. The most simple idea is a comparison of the final concept vectors of each agent in the CG with the concept vectors of the $\mathrm{LC}$ and of the $\mathrm{RC}$ and then to define a "political distance" by the scalar products of the previous concept vectors. However, as clarified in ref. [13], the choice of the concept vectors is not unique and only the subspace they span is relevant for the dynamical process. Then the criterion has to be related with the subspace spanned by CG agent concept vectors at the equilibrium with respect to the LC subspace (i.e. spanned by the concept vectors of the LC agent) or to the RC subspace and it is natural to consider the "angle", $\theta$, between two subspaces as the agent-agent distance [21].
The procedure is the following one. Firstly one considers the, $K, \gamma_{i}$ concept vectors of agent $i$ as columns of a new matrix $\Omega_{i}(D \times K$ dimensional matrix). Similarly we construct the matrix $\Omega_{j}$ of any agent of LC and of the RC (fixed by definition). According to refs. 22, 23], we calculate the overlap between $\Omega_{i}$ and $\Omega_{j}$ in the following way:

$$
\Omega_{i}^{\prime}=\Omega_{i}-\Omega_{j}\left(\Omega_{j}^{T} \Omega_{i}\right)
$$

Hence, we calculate the angle $\theta$ (in $[0, \pi / 2])$ as

$$
\theta_{i, L}=\arcsin \left(\min \left[1,\left\|\Omega_{i}^{\prime}\right\|_{\infty}\right]\right)
$$

and

$$
\theta_{i, R}=\arcsin \left(\min \left[0,\left\|\Omega_{i}^{\prime}\right\|_{\infty}\right]\right)
$$

where $\left\|\Omega_{i}^{\prime}\right\|_{\infty}$ is the biggest singular value of $\Omega_{i}^{\prime}$ according to the Singular Value Decomposition (SVD). With this definition the distance is between 0 (politically equivalent) and $\pi / 2$ (politically orthogonal). Finally the agent $i$ will vote for the $\mathrm{RC}$ or the $\mathrm{LC}$, according to the fact that the smallest "angle" is $\theta_{i, R}$ or $\theta_{i, L}$, respectively.

However, in order to have a more realistic model of the decision process, one has to take into account another important political aspect: the bipolar systems are, indeed, not perfectly bipolar. There is a non negligible part of the individuals in the CG that at the end of the dynamical evolution is still "too far" from the political ideas of the $\mathrm{L}$ and the $\mathrm{R}$ coalition and decide to vote for other possible groups (OG).

Of course one can neglect this point, which, however, is a crucial ingredient of the political dynamics.

Our definition of "political distance" between two agents is related to the angle $0<\theta<\pi / 2$ of the corresponding subspaces spanned by the concept vectors. When $\theta=\pi / 2$ two subspaces are orthogonal and then an agent $i$ of the CG will be considered voting for the OG if both the angles $\theta_{i, R}$ and $\theta_{i, L}$ are in the range between a fixed value $\epsilon$ and $\pi / 2$. An increase of $\epsilon$ decreases the number of agents in the CG who vote for the third group: $\epsilon$ represents the "mobility" of the CG toward the political coalitions.

\section{THE APPROXIMATIONS}

The equilibrium and dynamic properties depend on the interaction matrices $c_{i j}, l_{i j}, r_{i j}$. As a first approximation, one considers for the local interaction a mean-field political network with $c_{i j}=l_{i j}=r_{i j}=h$. This implies that, without global effects, the final voting choice is directly correlated with the number of agents of the LC and of the RC: a larger number of agents in the LC (RC) 
will automatically determine that a larger number of CG agents will we "closer" to the LC (RC).

Moreover, the global effects change the interaction strength of the agent $i$ in the CG with the voters of the $\mathrm{L}$ and $\mathrm{R}$ coalitions, by the satisfaction index $\delta_{i}$ (the interaction within the $\mathrm{CG}$ is unmodified).

For simplicity, let us assume that $\delta_{i}=\delta$ is independent on the agent $i$. By definition, it has opposite effect on the interactions with the $\mathrm{LC}$ and the $\mathrm{RC}$. Then the starting approximation for the world matrix $W_{i}$ of the agent $i$ in the CG, which includes the local and the global interactions, can be written as

$\boldsymbol{W}_{i}=\boldsymbol{W}_{i}^{0}+\sum_{j \in \mathcal{C} \mathcal{G}_{i}} h \boldsymbol{S}_{j}+\sum_{j \in \mathcal{L C} \mathcal{C}_{i}} h(1+\delta) \boldsymbol{S}_{j}+\sum_{j \in \mathcal{R} \mathcal{C}_{i}} h(1-\delta) \boldsymbol{S}_{j}$

Where $\delta$ has been conventionally assumed positive if the interaction with the $\mathrm{LC}$ increases. The parameter $\delta$ drives the opinion dynamics and determines the majority. A more detailed analysis of its meaning and effects is carried out in section 4 .

Another assumption concerns the initial core distributions of the $\mathrm{L}$ and the $\mathrm{R}$ coalitions. In fact, it is not difficult to take into account a numerical difference between the core voters of the two coalitions, but this would introduce another parameter in the model and, in the present work, we are mainly interested in analyzing the global effects on the vote decision. Therefore, in this first version of the model, we shall consider a symmetrical initial distribution for the core voters of the $\mathrm{L}$ and $\mathrm{R}$ coalitions. Moreover, abstentionism is not of dynamical origin and then, in the numerical simulation, is proportionally divided according to the group initial distributions.

The initial core voters of the $\mathrm{L}$ and $\mathrm{R}$ coalitions in our simulations are assumed equal to the $41 \%$ of valid votes while the CG has only the $18 \%$.

These numbers are not far from the real political situation: we verified that the lowest level for the $\mathrm{L}$ and $\mathrm{R}$ coalitions (assumed as the cores) in Italian national votes, from 1996 to 2004 , is close to the $41 \%$ of the valid votes.

This implies that the winning coalition is determined by the decision of a relatively small number of individuals of the CG.

\section{VOTE ANALYSIS}

Before analyzing the data of the national vote in Italy and in Germany, let us note that in the model there are essentially two parameters, $\delta$ and $\epsilon$, because, in the data fitting, a change in the parameter $h$ gives a rescaling of the previous ones.

For the simulations of the dynamical evolution one considers the initial configuration previously discussed, with
$\mathrm{D}=10, \mathrm{~K}=4$ and 10.000 agents. The results are averaged on 200 samples.

\section{Italian elections}

Table【reports the results of the Italian national vote from 1994 to 2004 for the different groups, the difference (in percent), $\Delta_{L R}$, in the final vote between the $\mathrm{L}$ and $\mathrm{R}$ coalitions and the fitted values of $\delta$ and $\epsilon$ which reproduce the data (starting with the initial configurations previously discussed).

Table I: The results of the Italian national vote from 1994 to 2004 for the three different groups (RC, LC, OG) and the difference $\left(\Delta_{L R}\right)$ (in percent). We also report the respective values of $\delta$ and $\epsilon$ which allow us to reproduce the data. The $\mathrm{L}$ coalition includes the following parties: Democratici di Sinistra, Margherita, Rifondazione Comunista, Verdi, Udeur, SDI, Italia dei valori, Comunisti Italiani. The R coalition contains Forza Italia, Alleanza Nazionale, Lega Nord, UDC, PRI. The other parties are in the third group.

\begin{tabular}{ccccccc}
\hline \hline Year & RC & LC & OG & $\Delta_{L R}$ & $\delta$ & $\epsilon$ \\
\hline 1994 & $50.4 \%$ & $45.3 \%$ & $4.3 \%$ & $-5.1 \%$ & -0.555 & 1.465 \\
1996 & $42.2 \%$ & $43.3 \%$ & $14.5 \%$ & $1.1 \%$ & 0.134 & 1.356 \\
1999 & $43.8 \%$ & $42.2 \%$ & $14.0 \%$ & $-1.6 \%$ & -0.195 & 1.361 \\
2001 & $49.5 \%$ & $46.3 \%$ & $4.2 \%$ & $-3.2 \%$ & -0.345 & 1.468 \\
2004 & $46.6 \%$ & $45.5 \%$ & $7.9 \%$ & $-1.1 \%$ & -0.121 & 1.425 \\
\hline \hline
\end{tabular}

The fit shows that when $\epsilon$ increases, the voters of the OG decrease and the final vote is politically more polarized: the difference between the two leading coalitions increases.

Moreover, when the (dis)satisfaction index $\delta$ is small also $\epsilon$ is small, i.e. the agents of the CG prefer to vote for a third group. In other words, when the consequences of the government choices are considered "neutral" by the CG agents, the latter tend not to vote for a more political coalition.

On the other hand, when the (dis)satisfaction increases, they feel that a stronger political option is more useful and vote for the $\mathrm{L}$ or the $\mathrm{R}$ coalition. There is anyway a "physiological" threshold of about $4 \%$ of voters who choose the OG.

The picture becomes more clear if one looks carefully the table and considers the evolution of the different Italian governments from 1994 to 2004.

In 1994 the RC won the election with a strong dissatisfaction toward the previous government $(\delta=-0.55$ and $\epsilon=1.465)$.

In 1996, due to the internal problem of the majority, a party left the RC and created an independent pole. This increased the $\mathrm{OG}$ and gave the final victory to the $\mathrm{LC}$ (by a moderate dissatisfaction $\delta=0.134$ and $\epsilon=1.36$ ). 
In 1999, the first negative signals for the LC government appeared as a small dissatisfaction $(\delta=-0.195)$ and a larger vote to the OG $(\epsilon=1.36)$ in the results of the election for the European Parliament.

In 2001 the national vote was polarized against the LC government $(\delta=-0.345$ and $\epsilon=1.47)$ and the $\mathrm{RC}$ won the election.

The European vote in 2004 is, in turn, a negative indication for the RC government because there is a strong reduction of $\delta(\delta=-0.12$ with respect to the previous value $\delta=-0.345$ ) and a clear signal of an emerging larger third group with respect to the previous election in 2001.

Hence the general behaviour of the agents in the CG seems to develop according the following steps: i) an initial political choice; ii) a dissatisfaction that leads to vote for the OG in the mean term elections; iii)a polarization against the Government in the following political election.

The previous considerations are probably obvious for an "educated" political observer and yet it is quite interesting that they are reproduced by a mathematical model. Moreover one can play a risky game: a prediction for the next Italian vote in April 2005 (see later).

\section{German elections}

Let us now consider the national vote in Germany from 1987 to 2002.

Since we also want to have indications about the different political behaviour of the Italian and German voters, we shall start with the previous initial distribution among the RC, the LC and the CG which, anyway, is not far from the minimum results obtained by the $\mathrm{R}$ and $\mathrm{L}$ coalitions (41.3\% and $39.7 \%)$.

Table II as table \before, reports the results of the German national vote for the different groups and the fitted values of $\delta$ and $\epsilon$.

Table II: As in tab. \the results of the German national vote from 1987 to 2002 for the three different groups (RC, LC, OG) and the difference $\left(\Delta_{L R}\right)$ (in percent). We also report the respective values of $\delta$ and $\epsilon$ which allow us to reproduce the data. The L coalition is given by SPD, Green party, PDS and the R coalition includes CDU, CSU and FDP. The other parties are in the third group.

\begin{tabular}{ccccccc}
\hline \hline Year & RC & LC & OG & $\Delta_{L R}$ & $\delta$ & $\epsilon$ \\
\hline 1987 & $53.4 \%$ & $45.3 \%$ & $1.3 \%$ & $-8.1 \%$ & -0.944 & 1.513 \\
1990 & $54.8 \%$ & $39.7 \%$ & $5.5 \%$ & $-15.1 \%$ & -2.215 & 1.410 \\
1994 & $48.3 \%$ & $48.1 \%$ & $3.6 \%$ & $-0.2 \%$ & -0.013 & 1.477 \\
1998 & $41.3 \%$ & $52.7 \%$ & $6.0 \%$ & $11.4 \%$ & 1.440 & 1.430 \\
2002 & $48.5 \%$ & $47.7 \%$ & $3.8 \%$ & $-0.8 \%$ & -0.080 & 1.475 \\
\hline \hline
\end{tabular}

There is a clear relation between $\epsilon$ and $\delta$ which shows that German voters have different attitude with respect to the Italian ones. Indeed, in this case, when $\delta$ increases $\epsilon$ decreases, i.e. the third group is larger. In other words, a bad government rating leads to vote for other groups rather than for the political coalitions. This is probably due to the fact that in Germany the other groups are essentially politically oriented while in Italy they tend to be more independent ones.

A deeper analysis requires, again, an understanding of the dynamics of abstentionism.

\section{The meaning of the variation of $\delta$}

The (dis)satisfaction index $\delta$ describes the rating of the government decisions as perceived by the agents and the parameter $\epsilon$ is a measure of the mobility of the CG.

With a positive/negative $\delta$ the majority will be of the $\mathrm{LC} / \mathrm{RC}$ coalition. However to have the majority of votes does not automatically mean that the corresponding coalition wins the election: a coalition which obtained a $60 \%$ majority in the last national vote and obtains only the $55 \%$ in the next one has still the majority (and then $\delta$ has the same sign) but with less consensus and, in this sense, does not win the election.

Therefore $\delta$ is effectively the index of the strength of the interaction of the $\mathrm{CG}$ with the $\mathrm{L}$ an $\mathrm{R}$ coalitions which determines the majority. Its variation from an election to the next one, $\Delta \delta$, is a more reliable "measure" of the evolution of the rating of the government and of the success of the coalitions.

The values of $\Delta \delta$ are obtained by table $\Delta \delta(99-96)=$ $-0.33, \Delta \delta(01-99)=-0.15, \Delta \delta(04-01)=0.22($ the elections before these dates are not considered because the victory of the LC in 1996 was due to a breaking of the $\mathrm{RC}$ and the government before the 94 election was a technical government rather than a political one) and they are correlated with the corresponding variations of $\epsilon, \Delta \epsilon$, as shown in fig. [1

The meaning of this correlation between $\Delta \delta$ and $\Delta \epsilon$ has been previously discussed and for the German vote one has a different behaviour with respect to the Italian case.

\section{A prediction: the next vote in Italy}

It is useful to show how the model can make some predictions and, as an example, let us consider the next Italian vote in April 2005.

For the prediction one needs to know the correlation between $\Delta \delta$ and $\Delta \epsilon$. In fig. 1 there are very few points and a reliable result requires a precise determination of the correlation. However, since it is useful, in our opinion, to show the procedure, let us consider in the numerical simulation the correlation obtained by fig. 1] 


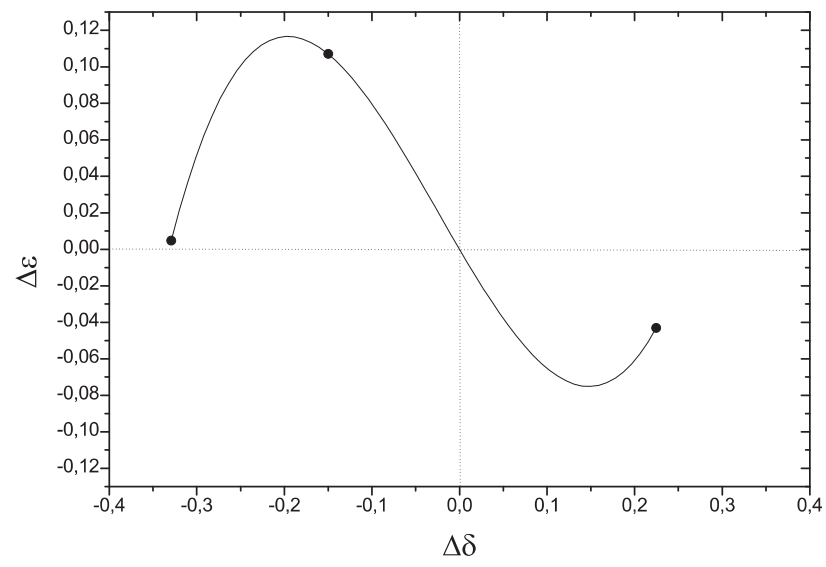

Figure 1: Correlation between $\Delta \delta$ and $\Delta \epsilon$ for the Italian case.

Unfortunately, the next Italian vote is not a national homogeneous ones but, rather, it is a regional election extended to the large part of the country (see later).

If one considers this election more politically homogeneous to the vote for the European Parliament, i.e. the crucial elements of the political debate are similar to the previous 2004 vote, therefore the starting point for the simulation is the election in 2004 and the results of the model as a function of $\Delta \delta(05-04)$ is reported in tab. III As in the previous analyses, abstentionism is fixed to $17 \%$.

Table III: The prediction of the model for the next Italian vote, when the 2004 election has been considered as the starting point.

\begin{tabular}{ccccc}
\hline \hline$\Delta \delta$ & $\Delta \epsilon$ & $\mathrm{RC}$ & $\mathrm{LC}$ & $\mathrm{OG}$ \\
\hline 0 & 0 & $46.6 \%$ & $45.4 \%$ & $8.0 \%$ \\
0.05 & -0.038 & $44.6 \%$ & $43.8 \%$ & $11.6 \%$ \\
0.10 & -0.065 & $43.1 \%$ & $42.8 \%$ & $14.1 \%$ \\
0.15 & -0.075 & $42.4 \%$ & $42.6 \%$ & $15.0 \%$ \\
0.20 & -0.061 & $42.8 \%$ & $43.5 \%$ & $13.7 \%$ \\
0.25 & -0.016 & $44.7 \%$ & $45.8 \%$ & $9.5 \%$ \\
0.30 & 0.066 & $47.2 \%$ & $48.8 \%$ & $4.0 \%$ \\
0.35 & 0.192 & $47.0 \%$ & $49.0 \%$ & $4.0 \%$ \\
0.40 & 0.367 & $46.7 \%$ & $49.3 \%$ & $4.0 \%$ \\
0.45 & 0.598 & $46.5 \%$ & $49.5 \%$ & $4.0 \%$ \\
0.50 & 0.891 & $46.3 \%$ & $49.7 \%$ & $4.0 \%$ \\
\hline \hline
\end{tabular}

On the other hand, if the next Italian vote is considered politically more similar to a national government election, the starting point of the analysis is the political result in 2001 and the prediction as a function of $\Delta \delta(05-01)$ are given in tab. IV

As one can see, worsening government rating, which implies a positive $\Delta \delta$, can cause today majority to loose the election and become minority. However, this depends on the numerical value of $\Delta \delta$ and the question arises if
Table IV: The prediction of the model for the next Italian vote, when the 2001 election has been considered as the starting point.

\begin{tabular}{ccccc}
\hline \hline$\Delta \delta$ & $\Delta \epsilon$ & $\mathrm{RC}$ & $\mathrm{LC}$ & $\mathrm{OG}$ \\
\hline 0 & 0 & $49.7 \%$ & $46.3 \%$ & $4.0 \%$ \\
0.05 & -0.038 & $47.7 \%$ & $44.8 \%$ & $7.5 \%$ \\
0.10 & -0.065 & $46.2 \%$ & $43.7 \%$ & $10.1 \%$ \\
0.15 & -0.075 & $45.5 \%$ & $43.5 \%$ & $11.0 \%$ \\
0.20 & -0.061 & $45.9 \%$ & $44.4 \%$ & $9.7 \%$ \\
0.25 & -0.016 & $47.7 \%$ & $46.7 \%$ & $5.6 \%$ \\
0.30 & 0.066 & $48.3 \%$ & $47.7 \%$ & $4.0 \%$ \\
0.35 & 0.192 & $48.0 \%$ & $48.0 \%$ & $4.0 \%$ \\
0.40 & 0.367 & $47.8 \%$ & $48.2 \%$ & $4.0 \%$ \\
0.45 & 0.598 & $47.6 \%$ & $48.4 \%$ & $4.0 \%$ \\
0.50 & 0.891 & $47.3 \%$ & $48.7 \%$ & $4.0 \%$ \\
\hline \hline
\end{tabular}

it is somehow related to the evolution of the opinions on the Government decisions as "detected", for example, by polls.

The answer to this risky question requires a careful comparison with the rating of the governments obtained by polls immediately before the various elections [24]. This point is interesting also for practical reasons (see sec. 5) and will be discussed in a forthcoming paper [25].

\section{CONCLUSIONS AND OUTLOOKS}

The proposed model is a first step towards a more realistic description of the opinion dynamics of the political vote and, indeed, it has many key elements of the decision process. However there are some approximations:

1) Abstentionism has not a dynamical origin.

This is an important point because the cores of the different coalitions do not change opinion, regardless of the value of the satisfaction index.

However, in the real political arena the winning coalition is determined not only by the vote decision of the agents in the CG but also by the decision not to vote of the agents in the core coalition: an agent with clear political convictions but strongly dissatisfied from his/her government does not vote for the opposite coalition but prefers the abstentionism. Since the winning coalition has only a few percent of vote more than the minority, this effect can easily determine the final result.

2) For the same reason,the symmetric distribution of the core of the $\mathrm{L}$ and $\mathrm{R}$ coalitions is an approximation: a small initially asymmetry of the coalitions can change the final result and/or increase the strength of the global parameter $\delta$ needed for the victory.

3) The symmetric mean field local interaction of the agents in the $\mathrm{CG}$ with the $\mathrm{LC}$ and $\mathrm{RC}$ individuals. 
Indeed, the opinion dynamics starts soon after a political vote and completes the evolution with the next vote choice. The agents in the CG who voted for the winning coalition are, at least immediately after the vote, predominately interested to interact with the majority. From this point of view, the symmetric local interaction means that the model applies for timeframe close to the next vote or, generally, when the agents of the CG become more independent respect to the previous majority. The points 1-3 require the introduction of (at least) other three parameters in the model.

Despite the previous approximations, that can be overcome by a more complete version, the model is able to:

a) describe the simplification procedure of the socialpolitical context;

b) introduce local and global interaction in the dynamics;

c) define a reliable "political distance" among the individuals;

d) introduce the alternative third choice for the voters;

e) determine phenomelogical relations among the parameters and some quantities obtained by data;

f) make some predictions;

g) potentially combine simulations and polls.

This last point is interesting because during the political polls, people are more incline to answer on more general and less direct political questions. For example, the answer is less uncertain if the question is about the coalition rather than the single political party. Now, it is not difficult to think of a set of undirect questions able to determine the parameters of the model $(\delta, \epsilon$ plus the others needed for points 1-3) and put on a more rigorous basis the relation among polls and mathematical models. Finally, the next steps include: a) a more realistic version of the model [25]; b) a combined effort with poll experts; c) the application of the model to different countries. Indeed, the correlation, if any, between $\delta$ and $\epsilon$ certainly depends on the peculiar characteristics of the considered nation but it should be important to verify if there are similar political behaviors in different places.

Acknowledgements. The authors thank G. Fath and D. Zappala' for useful suggestions and comments, S. Fortunato and G. Gambarelli for interesting discussions and R. Fonda, of the SWG, for the help in understanding the relation among the model parameters and the political poll results.

[1] W. Weidlich, Sociodynamics - A Systematic Approach to Mathematical Modelling in Social Sciences, Harwood Academic Publishers, 2000.
[2] D. Stauffer, The Monte Carlo Method on the Physical Sciences, J. E. Gubernatis ed., AIP Conf. Proc. 690147 (2003); "Sociophysics simulation II: opinion dynamics", physics/0503115

[3] S. Galam, J. Stat. Phys. 61,943 (1990); Physica A 238, 66 (1997).

[4] E. Bonabeau, G. Theraulaz and J. L. Deneubourg, Physica A 217, 373 (1995).

[5] B. Latane' and A. Nowak, in Progress in Communication Science, pp.43, G. A. Barnett and F. J. Boster eds. (1997).

[6] K. Sznajd-Weron and J. Sznajd, Int. J. Mod. Phys. C 11, 1157 (2000).

[7] R. Axelrod, J. Conflict Res. 41, 203 (1997) and The Complexity of Cooperation, Princeton University Press 1997.

[8] C. Castellano, M. Marsili and A. Vespignani, Phys. Rev. Lett 85, 3536 (2000).

[9] G. Deffuant, D. Neau, F. Amblard and G. Weisbuch, Adv. Compl. Syst. 3, 87 (2000); G. Weisbuch, G. Deffuant, F. Amblard and J. P. Nadal, Complexity 7,87 (2002); G. Deffuant, F. Amblard, G. Weisbuch and T. Faure, Journal of Artificial Societes and Social Simulations $\mathbf{5}$, issue 4 , (2002).

[10] R. Hegselmann and U. Krause, Journal of Artificial Societes and Social Simulations 5, issue 3, (2002).

[11] F. Vazquez and S. Redner, "Ultimate fate of constrained voters", cond-mat/0405652

[12] S. Fortunato, "The Sznajd consensus model with continuous opinions", cond-mat/0407353

[13] G. Fath and M. Sarvary, Physica A 348,611 (2005) and in "Economics and Heterogeneous Interacting Agents" Lecture notes in Economic and Mathematical Systems, A.Namate,T.Kaizouji and Y.Aruka eds, Springer (2005).

[14] G. Owen, Game Theory- Academic Press, San Diego 1995; for a review see G.Gambarelli and G.Owen, "The Coming of Game Theory", Essay on Cooperative Gamesin honour of G. Owen- Special Issues of Theory and Decision 36, 1 (2004),G.Gambarelli ed., Kluwe Academic Publishers.

[15] S. Fortunato, "Damage spreading and opinion dynamics on scale free networks", cond-mat/0405083

[16] S. Fortunato and D. Stauffer, "Computer simulation of opinions", cond-math/0501730

[17] S. R. White, Phys. Rev. Lett. 69, 2863 (1992).

[18] S. R. White, PRL 69, 2863 (1992), PRB 48, 10345 (1993).

[19] J. Wishart, Biometrica A 20, 32 (1928).

[20] A. Edelman, Ph.D. thesis, MIT (1989), downloadable from http://www-math.mit.edu/ ${ }^{\sim}$ edelman

[21] G. Fath, private communication.

[22] A. Bjorck \& G. Golub, Numerical methods for computing angles between linear subspaces, Math. Comp. 27 (1973), pp. 579-594.

[23] P. A. Wedin, On angles between subspaces of a finite dimensional inner product space, in B. Kagstrom \& A. Ruhe (Eds.), Matrix Pencils, Lecture Notes in Mathematics 973, Springer, 1983, pp. 263-285.

[24] R. Fonda, SWG Italy, private communications.

[25] F. Caruso and P. Castorina, in preparation. 